\title{
Effects of diets rich in plant polyphenols in mouse models of neurodegenerative disorders
}

Maria A. Tikhonova

Scientific Research Institute of

Physiology and Basic Medicine

Novosibirsk, Russia

tikhonovama@physiol.ru

Marina V. Ovsyukova

Scientific Research Institute of

Physiology and Basic Medicine

Novosibirsk, Russia

maryov@ngs.ru
Tamara G. Amstislavskaya

Scientific Research Institute of

Physiology and Basic Medicine

Novosibirsk, Russia

amstislavskaya@yandex.ru

Michael V. Tenditnik

Scientific Research Institute of

Physiology and Basic Medicine

Novosibirsk, Russia

m.v.tenditnik@physiol.ru
Anna A. Akopyan

Scientific Research Institute of Physiology and Basic Medicine

Novosibirsk, Russia annaaleksanovna@mail.ru

Elena K. Khlestkina

N.I. Vavilov All-Russian Research Institute of Plant Genetic Resources

St. Petersburg, Russia

Federal Research Center "Institute of

Cytology and Genetics"

Novosibirsk, Russia

khlest@bionet.nsc.ru

\begin{abstract}
Currently functional nutrition has developed intensively. Functional foods enriched with biologically active substances are an essential part of dietary therapy. Plant polyphenols attract particular attention due to their multiple beneficial properties. This study was aimed to evaluate the effects of diets rich in plant polyphenols in a mouse model of Alzheimer's disease (AD) induced by amyloid-beta and a transgenic model of Parkinson's disease (PD) in mice of B6.CgTg (Prnp-SNCA*A53T)23Mkle/J strain with overexpression of human alpha-synuclein. We used two wheat lines created at the Institute of Cytology and Genetics SB RAS [1] that have almost similar genomes with the exception of a small part of chromosome $2 \mathrm{~A}$, which contains a gene regulating anthocyanin biosynthesis, to assess the effects of an anthocyanin-rich grain diet, and a concentrate of grape polyphenols "Enoant" dissolved in the drinking water in addition to standard chow. Mice were kept at those diets for five months. Mice with AD model demonstrated deficits in working and short-term spatial memory. Both grain diets prevented the development of disturbances in working memory but not in short-term spatial memory. Mice of transgenic PD model also showed deficits in working memory that were improved only by the grain diet with high content of anthocyanins. The anthocyanin-rich grain diet
\end{abstract}

enhanced microglial activation and increased the expression of arginase1 marking the M2 microglia that promotes tissue viability and neuronal survival in the brain of transgenic PDlike mice. Although "Enoant" did not produce significant effects in AD and PD models, it substantially prolonged the average life span in mice with natural aging.

Keywords - functional food, wheat grain, anthocyanins, grape polyphenols, mice, Alzheimer's disease, amyloid-beta, alpha-synuclein, Parkinson's disease.

\section{ACKNOWLEDGMENT}

The study was supported by Russian Science Foundation (grant No. 16-14-00086).

\section{REFERENCES}

[1] E. I. Gordeeva, O. Y. Shoeva, and E. K.Khlestkina, "Marker-assisted development of bread wheat near-isogenic lines carrying various combinations of purple pericarp (Pp) alleles," Euphytica, vol. 203, pp. 469-476, 2015. 